\title{
Transparente Karriereplanung - Was entwickelt sich aus der Juniorprofessur?
}

DOI: $10.1007 / \mathrm{s} 12268-017-0788-2$

(C) Springer-Verlag 2017

Die Diskussion über nachhaltige Karrierewege in der Wissenschaft hat mit der Verabschiedung des Bund-Länder-Programms zur Förderung des wissenschaftlichen Nachwuchses im letzten Jahr neue Fahrt aufgenommen. In dessen Rahmen sollen in den nächsten Jahren 1.000 Tenure-Track-Stellen geschaffen werden, deren Finanzierung vom Bund für die ersten acht Jahre übernommen wird. Danach übernehmen die Länder die Finanzierung, sodass es dauerhaft zu einer entsprechenden Zahl an zusätzlichen Professuren kommen soll. Aus der Sicht der Deutschen Gesellschaft Juniorprofessur e. V. (DGJ) ist diese Entwicklung ausdrücklich zu begrüßen, weil ein Wandel hin zu einem auf Nachhaltigkeit ausgerichteten wissenschaftlichen Karrieresystem angestoßen wird.

Insbesondere im naturwissenschaftlichen Bereich erwarten wir auch direkte Auswirkungen auf die Produktivität. Ohne Tenure Track stehen Juniorprofessorinnen und -professoren oft unter einem erhöhten Druck, kurzfristige Erfolge zu erzielen, um sich möglichst schnell auf unbefristete Professuren bewerben zu können. In den Experimentalwissenschaften ist dies jedoch tendenziell mit Abstrichen an der Qualität verbunden, da anspruchsvolle Forschungsvorhaben typischerweise komplexe Laboraufbauten benötigen und somit eine lange Vorlaufzeit mit sich bringen. Eine Entfristungsperspektive hingegen kann es jungen Forscherinnen und Forschern ermöglichen, in ihren Projekten schon frühzeitig ambitioniertere, längerfristige Schwerpunkte zu setzen. Die Notwendigkeit, nach kurzer Zeit genügend Ergebnisse für eine Weiterbewerbung zu haben, würde entschärft.

Darüber hinaus führt eine Tenure-TrackPerspektive zu einer höheren Zufriedenheit. In einer Umfrage der DGJ unter ca. 500 Nachwuchswissenschaftlerinnen und -wissenschaftlern sagen nur 50 Prozent derer ohne Entfristungsperspektive, sie seien zufrieden mit der persönlichen und beruflichen Situation. Bei denen mit Tenure Track sind dies mit 64 Prozent deutlich mehr.

Hierbei spielt unserer Einschätzung nach eine wichtige Rolle, dass ein transparent ausgestalteter Tenure Track die Planbarkeit der Karriere erhöht. Bei der Bewertung der nun in das Auswahlverfahren des Bund-Länder-Programms eingebrachten Ausgestaltungsvorschläge der Universitäten sollte die Transparenz des Verfahrens daher unserer Meinung nach eine bedeutende Rolle spielen. Wie groß der diesbezügliche Handlungsbedarf ist, wird deutlich, wenn man als Vergleich die Zwischenevaluation bei der Juniorprofessur heranzieht. In der oben angesprochenen DGJ-Umfrage haben nämlich nur 38 Prozent derer, für die eine Zwischenevaluation vorgesehen ist bzw. war, den Evaluationsprozess und nur 36 Prozent die Evaluationskriterien als transparent beurteilt. Eine derart geringe wahrgenommene Transparenz darf es unserer Einschätzung nach in den Verstetigungsverfahren im Tenure Track nicht geben, da diese im Gegensatz zur Zwischenevaluation eine deutliche höhere Relevanz für die Evaluierten haben.

Dabei ist es sehr wichtig, den Besonderheiten der einzelnen Fachgebiete Rechnung zu tragen. Auch dies wird anhand unserer Umfrage deutlich, wiederum für die Zwischenevaluation: So nehmen im natur- und lebenswissenschaftlichen Bereich ca. 63 Prozent der Teilnehmerinnen und Teilnehmer das Einwerben von Drittmitteln als ein sehr wichtiges oder sogar äußerst wichtiges Kriterium der Zwischenevaluation wahr, während es unter den Befragten anderer Fächer nur von 46 Prozent entsprechend eingeschätzt wird. Ein ähnliches Bild ergibt sich bei der Relevanz von Preisen und Auszeichnungen (23 versus zehn Prozent). Soweit derartige Differenzen in der Wahrnehmung realen Unterschieden in den Erwartungen entsprechen, müssen die daraus resultierenden Einschränkungen der Vergleichbarkeit zwischen Disziplinen im Verfahren berücksichtigt werden. Ansonsten müssen die fachübergreifenden Erwartungen für eine erfolgreiche Tenure-Evaluation klar formuliert werden, um so Fehlwahrnehmungen entgegenzuwirken und den Tenure-Track-Professorinnen und -professoren eine akkurate Einschätzung ihres Leistungsstandes zu ermöglichen. Nur mit durchgehender Transparenz des Verfahrens kann die Planbarkeit der wissenschaftlichen Karriere erhöht und durch einen ernsthaften Evaluationsprozess eine hohe Qualität in Lehre und Forschung sichergestellt werden.

Wenn seitens der antragstellenden Hochschulen die Bereitschaft vorhanden ist, entsprechend attraktive Tenure-Track-Konzepte zu entwickeln und die Tenure-Track-Professuren adäquat in ihre Hochschullehrerschaft zu integrieren, besteht unserer Meinung nach die Chance, dass das Bund-Länder-Programm zu einem Erfolg wird und so den Weg für eine dauerhafte Weiterentwicklung des deutschen Wissenschaftssystems hin $\mathrm{zu}$ nachhaltiger Karriereplanung ebnet.

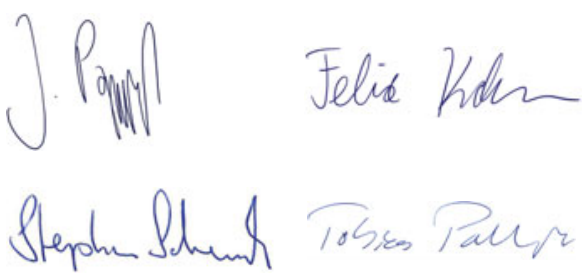

Jens Pöppelbuß, Felix Krahmer, Stephan Scherneck, Tobias Potthoff, Deutsche Gesellschaft Juniorprofessur e. V.

\section{Korrespondenzadresse:}

Deutsche Gesellschaft Juniorprofessur e. V. c/o Prof. Dr. Jens Pöppelbuß

Kriegerstraße 27

D-26123 Oldenburg

Tel.: 0160-96951103

info@juniorprofessur.org

www.juniorprofessur.org

Das Karriereportal der ZEIT, www.academics.de, erklärt Tenure-Track: „Der Tenure Track ist dem amerikanischen Bildungssystem entlehnt und ist seit der Novellierung des Hochschulrahmengesetzes 2002 ein möglicher Zusatz bei der Ausschreibung einer Juniorprofessur. Tenure Track bedeutet hierbei die Zusage nach einer befristeten Bewährungszeit eine Lebenszeitprofessur zu erhalten."

Jens Pöppelbuß ist Juniorprofessor für Industrienahe Dienstleistungen an der Universität Bremen sowie Vorstandsvorsitzender der Deutschen Gesellschaft Juniorprofessur e. V. (DGJ).

Felix Krahmer ist Assistant Professor für Optimierung und Datenanalyse an der TU München sowie Schriftführer und Kassenwart der DGJ.

Stephan Scherneck ist Juniorprofessor am Institut für Pharmakologie, Toxikologie und Klinische Pharmazie der TU Braunschweig sowie stellvertretender Vorstandsvorsitzender der DGJ.

Tobias Potthoff ist wissenschaftlicher Mitarbeiter an der Universität Duisburg-Essen, Promotionsstipendiat der Stiftung der Deutschen Wirtschaft und Geschäftsführer der DGJ. 